\title{
Calculations of Integrals of Products of Bessel Functions
}

\author{
By J. E. Kilpatrick, ${ }^{1}$ Shigetoshi Katsura ${ }^{2}$ and Yuji Inoue ${ }^{3}$
}

I. Introduction. Integrals of products of Bessel functions are of general interest. Define $W$-integrals by

$$
\begin{aligned}
W_{\nu_{1} \nu_{2} \cdots \nu_{l}}^{\lambda} & \left(a_{1}, a_{2} \cdots a_{l}\right) \\
& \equiv \int_{0}^{\infty} J_{\nu_{1}}\left(a_{1} t\right) J_{\nu_{2}}\left(a_{2} t\right) \cdots J_{\nu_{l}}\left(a_{l} t\right) t^{-\lambda} d t .
\end{aligned}
$$

In this note we consider

$$
W_{3 / 2+n_{1}, 3 / 2+n_{2}, 3 / 2+n_{3}, 3 / 2+n_{4}}^{4}(1,1,1,1) \equiv U_{1}
$$

where $n_{1}, n_{2}, n_{3}, n_{4}$ are zero or positive integers and $n_{1}+n_{2}+n_{3}+n_{4}$ is even, and

$$
W_{3 / 2+n_{1}, 3 / 2+n_{2}, 3 / 2+n_{3}}^{5 / 2}(a, b, b) \equiv U_{2},
$$

where $n_{1}, n_{2}, n_{3}$ are zero or positive integers, $n_{1}+n_{2}+n_{3}$ is even and $a, b$ are real positive numbers. The symbols $U_{i}$ are used for convenient reference.

Integrals (1.1) and (1.2) together with integrals of threefold and fourfold products of associated Legendre functions [7], were used in the calculation of virial coefficients in statistical mechanics [3], [4], [5], [6].

The usual numerical integration techniques such as Simpson's method, Gauss' method, method of indefinite integral of polynomials [7] etc., when applied to integrals with oscillating integrands such as in (1.1) and (1.2) are inefficient.

Values of integrals (1.1) and (1.2) can be obtained by transforming them into Mellin-Barnes integrals [1], [2], etc., or Meijer's $G$-functions [2], and application of the residue calculus as developed by one of the authors in [3] leads to the exact determination of the integrals.

As the Mellin-Barnes integrands are rather complicated, one has to do considerable scanning to determine the actual poles. In this paper, the scanning process and the evaluation of the residues at these poles is computer programmed.

II. $W_{3 / 2+n_{1}, 3 / 2+n_{2}, 3 / 2+n_{3}, 3 / 2+n_{4}}^{4}(1,1,1,1)$. We consider the integral (1.1).

We use a series representation of the products of the two Bessel functions [9]:

Received December 16, 1966. Revised February 3, 1967.

1 Department of Chemistry, Rice University, Houston, Texas.

${ }^{2}$ Department of Applied Physics, Tôhoku University, Sendai, Japan.

${ }^{3}$ Research Institute for Electrical Communication, Tôhoku University, Sendai, Japan. 


$$
\begin{aligned}
& J_{3 / 2+n_{1}}(t) J_{3 / 2+n_{2}}(t) \\
& =\sum_{s=0}^{\infty} \frac{(-)^{s}\left(\frac{1}{2} t\right)^{3 / 2+n_{1}+3 / 2+n_{2}+2 s}\left(\frac{3}{2}+n_{1}+\frac{3}{2}+n_{2}+2 s+1\right)}{s ! \Gamma\left(\frac{3}{2}+n_{1}+s+1\right) \Gamma\left(\frac{3}{2}+n_{2}+s+1\right) \Gamma\left(\frac{3}{2}+n_{1}+\frac{3}{2}+n_{2}+s+1\right)} \\
& =\frac{1}{\sqrt{ } \pi} \sum_{s=0}^{\infty} \frac{(-)^{s n_{1}+n_{2}+3+2 s} \Gamma\left(s+\frac{n_{1}+n_{2}+4}{2}\right) \Gamma\left(s+\frac{n_{1}+n_{2}+5}{2}\right)}{s ! \Gamma\left(s+n_{1}+\frac{5}{2}\right) \Gamma\left(s+n_{2}+\frac{b}{2}\right) \Gamma\left(s+n_{1}+n_{2}+4\right)} . \\
& \equiv U_{3} \text {. }
\end{aligned}
$$

The summation over $s$ is replaced by a complex integral over $d s$ by using the fact that the residue of $\Gamma(z)$ at $z=-l$ is $(-)^{l} / l$ ! for a zero or positive integral value of $l$. Hence

$$
U_{3}=\frac{1}{\sqrt{ } \pi} \frac{1}{2 \pi i} \int_{s_{0}-i \infty}^{s_{0}+i \infty} \frac{\Gamma(-s+A) \Gamma(s+E) \Gamma(s+F) t^{n_{1}+n_{2}+2 s+3}}{\Gamma(s+L) \Gamma(s+J) \Gamma(s+K)},
$$

where

$$
\begin{gathered}
A=0, \quad L=n_{1}+5 / 2, \quad E=\left(n_{1}+n_{2}+4\right) / 2, \quad J=n_{2}+5 / 2, \\
F=\left(n_{1}+n_{2}+5\right) / 2, \quad K=n_{1}+n_{2}+4,
\end{gathered}
$$

where $s_{0}$ is chosen in such a way that the poles of $\Gamma(-s+A)$ lie on the right of the path and the poles of $\Gamma(s+E)$ and $\Gamma(s+F)$ on the left of the path.

From $(2.4)-F<-E$, thus

$$
-E<s_{0}<A .
$$

Substituting (2.3) into (1.1), we have

$$
\begin{aligned}
U_{1}= & \frac{1}{\sqrt{ } \pi} \int_{0}^{\infty} d t \frac{1}{2 \pi i} \int_{s_{0}-i \infty}^{s_{0}+i \infty} \frac{\Gamma(-s+A) \Gamma(s+E) \Gamma(s+F)}{\Gamma(s+L) \Gamma(s+J) \Gamma(s+K)} \\
& \times J_{3 / 2+n_{3}}(t) J_{3 / 2+n_{4}}(t) t^{n_{1}+n_{2}+2 s+3} d s \equiv U_{4} .
\end{aligned}
$$

Next, $s_{0}$ is moved to the left in such a way that the condition for the convergence of Weber-Schafheitlin's integral [9], [2]

$$
\int_{0}^{\infty} J_{3 / 2+n_{3}}(t) J_{3 / 2+n_{\mathbf{4}}}(t) t^{-1+n_{1}+n_{2}+2 s} d t
$$

is satisfied, if necessary. That is, $s_{0}$ is chosen to satisfy simultaneously (2.5) and

$$
-D<s_{0}<B
$$

where

$$
D=\frac{3+n_{1}+n_{2}+n_{3}+n_{4}}{2}, \quad B=\frac{1-n_{1}-n_{2}}{2} .
$$

Then, the order of the integration $\int d t$ and $\int d s$ can be changed, and the integration over $d t$ yields the Mellin-Barnes integral [1], [2]: 


$$
\begin{aligned}
U_{4}= & \frac{1}{\sqrt{ } \pi} \frac{1}{2 \pi i} \int_{s_{0}-i \infty}^{s_{0}+i \infty} \frac{\Gamma(-s+A) \Gamma(s+E) \Gamma(s+F)}{\Gamma(s+J) \Gamma(s+K) \Gamma(s+L)} d s \\
& \times \int_{0}^{\infty} J_{3 / 2+n_{3}}(t) J_{3 / 2+n_{4}}(t) t^{-1+n_{1}+n_{2}+2 s} d t \\
= & \frac{1}{2 \pi} \frac{1}{2 \pi i} \int_{s_{0}-i_{\infty}}^{s_{0}+i_{\infty}} \\
& \times \frac{\Gamma(-s+A) \Gamma(-s+B) \Gamma(-s+C) \Gamma(s+D) \Gamma(s+E) \Gamma(s+F)}{\Gamma(-s+G) \Gamma(-s+H) \Gamma(-s+I) \Gamma(s+J) \Gamma(s+K) \Gamma(s+L)} d s \\
\equiv & U_{5},
\end{aligned}
$$

where

$$
\begin{array}{ll}
C=\frac{2-n_{1}-n_{2}}{2}, & G=\frac{2-n_{1}-n_{2}-n_{3}+n_{4}}{2}, \\
H=\frac{5-n_{1}-n_{2}+n_{3}+n_{4}}{2}, & I=\frac{2-n_{1}-n_{2}+n_{3}-n_{4}}{2} .
\end{array}
$$

The right-hand side of (2.11) belongs to the third type of Mellin-Barnes integrals [1], [2], in which the complex variable $z$ is set equal to 1 . Hence it is expressed as a Meijer $G$-function [2].

$$
U_{5}=\frac{1}{2 \pi} G_{66}^{33}\left(1 \mid \begin{array}{l}
1-D, 1-E, 1-F ; G, H, I \\
A, B, C ; 1-J, 1-K, 1-L
\end{array}\right) .
$$

Because of the interrelationships of the quantities $A, B, \cdots, L$, the above $G$ function cannot be expanded in the usual fashion as a sum of three generalized hypergeometric functions of the form ${ }_{6} F_{5}$. However, limiting forms of the general expansion can be taken. In this connection, see [8, p. 14]. In the present instance, the resultant expressions depend on the interrelations between $n_{1}, n_{2}, n_{3}$ and $n_{4}$. Thus, to avoid any specific configurations among the $n_{i}$, we evaluate the second integral representation in $(2.10)$ by using the poles like $\Gamma(-s+A)$ which lie to the right of the path of integration.

III. Programing for Computers. We define $G(s) b^{4}$

$$
\begin{array}{r}
G(s)=\Gamma(s), \text { when } s \text { is a positive integer, } \\
\text { or a positive or negative half odd integer, } \\
G(s)=\frac{(-)^{-s}}{(-s) !}, \begin{array}{l}
\text { when } s \text { is zero or a negative integer, } \\
(G(s) \text { is residue of } \Gamma(s)) .
\end{array}
\end{array}
$$

Then (2.11) is given by

$$
\begin{aligned}
U_{5} & =\frac{1}{2 \pi} \sum_{\text {poles }} \frac{G(-s+A) G(-s+B) G(-s+C) G(s+D) G(s+E) G(s+F)}{G(-s+G) G(-s+H) G(-s+I) G(s+J) G(s+K) G(s+L)} \\
& \equiv U_{6},
\end{aligned}
$$

${ }^{4}$ Meijer $G$-functions and $G(s)$ defined here should not be confused. 
where $\sum_{\text {poles }}$ means a sum over poles of integrand of $(2.11)$ on the right of the path of the integration.

The value of $s$ which can be a pole is an integer or a half odd integer. When the value of an argument of a $G$-function in the numerator, say, $-s+A$, is a negative integer or zero for some integral or half odd integral value of $s$, a weight factor +1 is given to the corresponding $G$-function. When the value in the denominator, say, $s+L$, is a negative integer or zero, a factor -1 is given to the corresponding $G$-function. Determine weight factors for all $G$-functions in the numerator and in the denominator.

When the sum of weight factors of $G$-functions in the numerator and those in the denominator is equal to +1 for a value (integer or half odd integer) of $s$, then $s$ is a simple pole. When it is equal to a positive integer $n, s$ is a $n$-ple pole. When it is equal to zero or a negative integer, $s$ is not a pole. In our example, double and higher-order poles do not appear. ${ }^{5}$

The values of $G(-s+A), G(-s+B), \cdots, G(s+L)$, are picked out from a table of $G(t)$ tabulated in a memory, and the values are substituted into (3.3); then it gives the residue of (2.11) at $s$. This process is repeated for every integral and half odd integral value of $s$ from $s_{\min }$ to $s_{\max }$, where $s_{\min }$ and $s_{\max }$ are the minimum and the maximum values of $s$ which can be a pole, respectively. ${ }^{6}$ They are given by

$$
s_{\min }=\operatorname{Min}(A, B), \quad s_{\max }=\operatorname{Max}(G, H, I) .
$$

Since the maximum value of $G(s)$ and the value of products of $G(s)$ in the course of the calculation sometimes cause overflow or underflow, the table of $\log |G(s)|$ is tabulated in the memory in the actual calculation. With regard to the sign of $G(s)$, $\mathrm{sg}(s)$ is defined for integral or half odd integral values of $s$ as follows,

$$
\begin{aligned}
& \operatorname{sg}(s)=-1, \quad(G(s)<0), \quad \text { for } s<0 \text { and }|2 s|=1 \text { or } 2(\bmod 4) \text {, } \\
& \operatorname{sg}(s)=+1, \quad(G(s)>0), \text { otherwise. }
\end{aligned}
$$
by

Then, after each factor of log $|G(s)|$ is added or subtracted, the sign is associated

$$
\begin{aligned}
U_{6}= & \frac{1}{2 \pi} \sum_{\text {poles }} \operatorname{sg}(-s+A) \operatorname{sg}(-s+B) \operatorname{sg}(-s+C) \operatorname{sg}(s+D) \\
& \times \operatorname{sg}(s+E) \mathrm{sg}(s+F) \operatorname{sg}(-s+G) \operatorname{sg}(-s+H) \operatorname{sg}(-s+I) \\
& \times \operatorname{sg}(s+J) \operatorname{sg}(s+K) \operatorname{sg}(s+L) \\
& \times \exp [\log |G(-s+A)|+\log |G(-s+B)|+\log |G(-s+C)| \\
& +\log |G(s+D)|+\log |G(s+E)|+\log |G(s+F)| \\
& -\log |G(-s+G)|-\log |G(-s+H)|-\log |G(-s+I)| \\
& \quad-\log |G(s+J)|-\log |G(s+K)|-\log |G(s+L)|] .
\end{aligned}
$$

5 When $n_{1}+n_{2}=$ even, all poles of $\Gamma(-s+C)$ are canceled by those of $\Gamma(-s+G)\left(n_{3} \geqq n_{4}\right)$ or of $\Gamma(-s+I)\left(n_{3} \leqq n_{4}\right)$. When $n_{1}+n_{2}=$ odd, all poles of $\Gamma(-s+B)$ are canceled by those of $\Gamma(-s+G)\left(n_{3}-n_{4}>-1\right)$ or of $\Gamma(-s+I)\left(n_{3}-n_{4} \leqq-1\right)$. Poles of $\Gamma(-s+A)$ and $\Gamma(-s+B)$ do not coincide.

${ }^{6}$ Poles of $\Gamma(-s+A)$ are canceled by those of $\Gamma(-s+I)$ for $I \leqq s\left(n_{3} \geqq n_{4}\right)$ or of $\Gamma(-s+G)$ for $G \leqq s\left(n_{3} \leqq n_{4}\right)$. When $n_{1}+n_{2}=$ even, poles of $\Gamma(-s+B)$ are canceled by those of $\Gamma(-s+H)$ for $H \leqq s$. When $n_{1}+n_{2}=$ odd, poles of $\Gamma(-s+C)$ are canceled by those of $\Gamma(-s+H)$ for $H \leqq s$. Hence, the number of poles is finite. 
A program to calculate (1.1) was made using the method described in this section.

IV. $W_{3 / 2+n_{1}, 3 / 2+n_{2}, 3 / 2+n_{3}}^{5 / 2}(a, b, b)$. A similar argument can be carried out for the integrals of three-fold products of Bessel functions (1.2) by substituting the integral representation for the Bessel function

$$
J_{3 / 2+n_{1}}(a t)=\frac{1}{2 \pi i} \int_{s_{0}-i \infty}^{s_{0}+i \infty} \frac{\Gamma(-s)}{\Gamma\left(5 / 2+n_{1}+s\right)}\left(\frac{a t}{2}\right)^{3 / 2+n_{1}+2 s} d s
$$

into the first factor of (1.2), where

$$
s_{0}<0
$$

The integral (1.2) is shown to satisfy

$$
\begin{aligned}
& U_{2}= \frac{1}{2} \cdot \frac{1}{\sqrt{ } \pi} b^{3 / 2}\left(\frac{a}{2 b}\right)^{3 / 2+n_{1}}-\frac{1}{2 \pi i} \int_{s_{0}-i \infty}^{s_{0}+i \infty} d s\left(\frac{a}{2 b}\right)^{2 s} \\
& \times \frac{\Gamma(-s+A) \Gamma(-s+M) \Gamma(-s+N) \Gamma(s+P)}{\Gamma(-s+Q) \Gamma(-s+R) \Gamma(-s+T) \Gamma(s+L)} \equiv U_{7}, \\
&-P<s_{0}<M,
\end{aligned}
$$

where

$$
\begin{array}{ll}
A=0, & Q=\frac{2-n_{1}-n_{2}+n_{3}}{2}, \\
M=\frac{1-n_{1}}{2}, & R=\frac{5-n_{1}+n_{2}+n_{3}}{2}, \\
N=\frac{2-n_{1}}{2}, & T=\frac{2-n_{1}+n_{2}-n_{3}}{2}, \\
P=\frac{3+n_{1}+n_{2}+n_{3}}{2}, & L=\frac{5+2 n_{1}}{2} .
\end{array}
$$

The right-hand side of (4.3) belongs to the third type of Mellin-Barnes integrals in which the complex variable $z$ is put to be equal to $(a / 2 b)^{2}$. Hence, the value of the integral can be obtained by calculating the sum of residues of the integrand at the poles of $\Gamma(-s+A), \Gamma(-s+M)$ and $\Gamma(-s+N)$ to the right of the path of integration when $a \leqq 2 b$, and those at the poles of $\Gamma(s+P)$ to the left of the path of integration when $a \geqq 2 b$. In particular $W_{3 / 2,3 / 2+n_{2}, 3 / 2+n_{3}}^{5 / 2}(2,1,1)$ vanishes for $n_{1}=0$ and $n_{2}+n_{3}$ (even) $\geqq 2$, since no poles are found to the left of the path of integration. Hereafter we consider the case where $a \leqq 2 b$.

The value $s_{0}$ must satisfy the condition of (4.2) and the convergence condition of the Weber-Schafheitlin's integral (4.4), simultaneously. Hence

$$
\begin{aligned}
U_{7}= & \frac{1}{4(2 \pi)^{1 / 2}} a^{3 / 2}\left(\frac{a}{2 b}\right)^{n_{1}} \sum_{\text {poles }}\left(\frac{a}{2 b}\right)^{2 s} \\
& \times \frac{G(-s+A) G(-s+M) G(-s+N) G(s+P)}{G(-s+Q) G(-s+R) G(-s+T) G(s+L)} \equiv U_{8}, \\
& s_{\min }=\operatorname{Min}(A, M), \quad s_{\max }=\operatorname{Max}(Q, R, T) .
\end{aligned}
$$


The right-hand side of (4.6) is expressed as

$$
U_{8}=\frac{1}{2 \sqrt{ } \pi} b^{3 / 2}\left(\frac{a}{2 b}\right)^{3 / 2+n_{1}} G_{44}^{31}\left(\left(\frac{a}{2 b}\right)^{2}\left(\begin{array}{l}
1-P ; Q, R, T \\
A, M, N ; 1-L
\end{array}\right) .\right.
$$

Summation in (4.6) is to be carried out by the same method as the fourfold products of Bessel functions.

V. Tables. Tables of integrals

$$
W_{3 / 2+n_{1}, 3 / 2+n_{2}, 3 / 2+n_{3}, 3 / 2+n_{4}}^{4}(1,1,1,1)
$$

for $n_{1}, n_{2}, n_{3}, n_{4} \leqq 10, n_{1},+n_{2}+n_{3}+n_{4}=$ cven, of

$$
W_{3 / 2+n_{1}, 3 / 2+n_{2}, 3 / 2+n_{3}}^{5 / 2}(a, b, b)
$$

for $n_{1}, n_{2}, n_{3} \leqq 20, n_{1}+n_{2}+n_{3}=$ even, $a, b, c=1$ and of (5.2) for $n_{1}=0, n_{2}, n_{3}$ $\leqq 16, n_{2}+n_{3}=$ even, $a, b, c=1$ or 2 were calculated by the present method and a copy was deposited as a document in the UMT file. ${ }^{7}$

Integrals $W_{\nu_{1} \nu_{2} \nu_{3}}^{\lambda_{1}}(a, b, b)$ and $W_{\nu_{1} \nu_{2} \nu_{3} \nu_{4}}^{\lambda_{4}}(a, a, b, b)$ for integral values of $\nu_{i}$ can be obtained by modifying the present method in such way that residues at double poles can be calculated. This can be done by storing $\Psi(n)$ and $\Psi\left(n+\frac{1}{2}\right)(\Psi(z)=$ $\left.\Gamma^{\prime}(z) / \Gamma(z)\right)$ in the memory.

Integrals $W_{\nu_{1} \nu_{2} \nu_{3}}^{\lambda}(a, b, c)$ and $W_{\nu_{1} \nu_{2} \nu_{3} \nu_{4}}^{\lambda}(a, b, c, d)$ for different $a, b, c, d$ are outside the range of applicability of the present method. They can be evaluated by the method of Appendix III in [3] for half integral values of $\nu_{i}$. General results along these lines will be reported in the future.

7 "Tables of integrals of products of Bessel function" by J. E. Kilpatrick, S. Katsura and Y. Inoue, UMT file number 27, Math. Comp., v. 21, p. 267. Numerical calculations were carried out by using the Rice University Computer, an IBM 7090 in UNICON (University Contribution of Japan IBM) and HITAC 5020 in Tokyo University. The authors acknowledge support from these computing centers.

1. A. L. Dixon \& W. L. Ferrar, "A class of discontinuous integrals," Quart. J. Math., v. 7 , 1936, pp. 81-96.

2. A. Erdélyi, W. Magnus, F. Oberhettinger \& F. G. Tricomi, Higher Transcendental Functions, Vol. 1, McGraw-Hill, New York, 1953, pp. 49, 206. MR 15, 419.

3. S. KATSURA, "Fourth virial coefficient for the square well potential," Phys. Rev., v. 115, 1959 , pp. $1417-1426$; v. 118, 1960, p. 1167. MR 21 \#6736.

4. S. KATSURA, "Fourth virial coefficient for the square well potential. II," J. Chem. Phys. v. 45,1966 , pp. $3480-3482$.

5. S. KatSURA \& Y. Abe, "Irreducible cluster integrals of hard sphere gases," J. Chem. Phys., v. 39,1963 , pp. $2068-2080$.

6. J. E. Kilpatrick \& S. Katsura, "Irreducible cluster integrals of hard sphere gases. II," J. Chem. Phys., v. 45, 1966, pp. 1866-1874.

7. S. Katsura, Y. Inoue, S. Yamashita \& J. E. Kilpatrick, "Tables of integrals of threefold and fourfold products of associated Legendre functions," Tech. Rep. Tôhoku Univ., v. 30, 1965, pp. 93-164. MR 33 \#309.

8. Y. L. LuKE, Integrals of Bessel Functions, McGraw-Hill, New York, 1962, p. 331. MR 25 \#5198.

9. G. N. WATson, A Treatise on the Theory of Bessel Functions, Cambridge Univ. Press, Cambridge; Macmillan, New York, 1922, pp. 147, 408; 2nd ed., 1944. MR 6, 64. 